\title{
Piezoresistive Sensing of Bistable Micro Mechanism State
}

Jeffrey K. Anderson

jeffanderson@byu.net

Larry L. Howell

Ihowell@byu.edu

Jonathan W. Wittwer

Timothy W. McLain

mclain@byu.edu

Follow this and additional works at: https://scholarsarchive.byu.edu/facpub

Part of the Mechanical Engineering Commons

\section{Original Publication Citation}

Anderson, J.K., Howell, L.L., Wittwer, J.W., and McLain, T.W., "Piezoresistive Sensing of Bistable Micro Mechanism State," Journal of Micromechanics and Microengineering, Vol. 16, No. 5, pp. 943-95, 26.

\section{BYU ScholarsArchive Citation}

Anderson, Jeffrey K.; Howell, Larry L.; Wittwer, Jonathan W.; and McLain, Timothy W., "Piezoresistive Sensing of Bistable Micro Mechanism State" (2014). Faculty Publications. 69.

https://scholarsarchive.byu.edu/facpub/69 accepted for inclusion in Faculty Publications by an authorized administrator of BYU ScholarsArchive. For more information, please contact ellen_amatangelo@byu.edu. 


\title{
Piezoresistive sensing of bistable micro mechanism state
}

\author{
Jeffrey K Anderson ${ }^{1}$, Larry L Howell ${ }^{1 *}$, Jonathan W Wittwer ${ }^{2}$, and Timothy W McLain ${ }^{1}$ \\ ${ }^{1}$ Department of Mechanical Engineering, Brigham Young University, 435 CTB, Provo, UT 84602, USA \\ ${ }^{2}$ Sandia National Laboratories, P.O. Box 5800, MS1310, Albuquerque, NM 87185-1310, USA \\ "Corresponding Author: Email: 1howell@byu.edu, Telephone: (801) 422-8037
}

\begin{abstract}
The objective of this work is to demonstrate the feasibility of on-chip sensing of bistable mechanism state using the piezoresistive properties of polysilicon, thus eliminating the need for electrical contacts. Changes in position are detected by observing changes in resistance across the mechanism. Sensing the state of bistable mechanisms is critical for various applications, including high-acceleration sensing arrays and alternative forms of nonvolatile memory. A fully compliant bistable micro mechanism was designed, fabricated, and tested to demonstrate the feasibility of this sensing technique. Testing results from two fabrication processes, SUMMiT IV and MUMPs, are presented. The SUMMiT mechanism was then integrated into various Wheatstone bridge configurations to investigate their potential advantages and to demonstrate various design layouts. Repeatable and detectable results were found with independent mechanisms and with those integrated into Wheatstone bridges.
\end{abstract}




\section{Introduction}

The objective of this work is to demonstrate the feasibility of on-chip sensing of bistable mechanism state using the piezoresistive properties of polysilicon. Two approaches are proposed and demonstrated: 1) sensing the resistance change in the mechanism caused by the strain in the second equilibrium position, and 2) integrating the mechanism in bridge configurations. Four different bridge configurations are investigated. Sensing the state of bistable mechanisms is critical in their various applications, such as mechanical nonvolatile memory or high-acceleration sensing arrays. The proposed approach allows on-chip state sensing with the potential of a dramatic increase in reliability.

Electrical contacts are often used to determine the state of a mechanism by using the mechanism to close an electrical circuit in one of its states. However, tolerances and fabrication variation lead to challenges with electrical contacts. Contact resistances can vary across a wafer and change between operating cycles. Polysilicon is a poor contact material because it is too hard, and its resistivity is too high. This work proposes piezoresistive position sensing to eliminate reliability issues and errors associated with electrical contacts by completely eliminating the contact. On-chip sensing is integrated into the device design, and low power is required to sense changes in mechanism position.

Measurable changes in resistance are required to detect the change in mechanism state, and several challenges exist that complicate the problem. The magnitude of the resistance change is dependent on device design, material properties, and fabrication process. The largest challenge is related to the fact that the sign of the resistance change in the mechanism is dependent on the direction of stress (tensile or compressive). Many MEMS devices use the piezoresistive effect on one surface of a membrane, which will be in tension or compression when under pressure. 
However, a beam in bending experiences both tensile and compressive stresses. The different signs of resistance change for compressive and tensile strain can combine to minimize the output signal or eliminate any detectable piezoresistive effect. Several devices are presented that address this challenge and make it feasible to use the piezoresistive properties of a bistable device to detect its state.

Testing on bistable mechanisms manufactured in SUMMiT IV process and MUMPs is presented. Four bridge configurations were then tested to investigate potential advantages. Models predicting the trends of resistive behaviors are also presented.

\section{Background}

A bistable mechanism is a device that can toggle between two stable equilibrium positions [1]. Compliant bistable mechanisms can be used in many applications including switches [2], valves [3], and relays [4]. The application of compliant micro bistable mechanisms is of interest because the mechanism will remain in either position without requiring input power to maintain the position.

Opdahl [5] presents a classification method which allows the characterization of various bistable mechanisms. Snap-through buckling mechanisms are well-suited for piezoresistive position sensing because there are no breaks in electric contact and there is a large difference in stress present in the mechanism between the first equilibrium position and a stopped, second stable position. A stop is used to increase the amount of stress in the second stable position, enabling a larger stress difference between the two measured positions.

Figure 1 shows a plot of the applied force vs. displacement curve for a bistable mechanism. This curve is the derivative of the energy curve with respect to displacement. The roots of the curves represent the peaks and valleys of energy, or the stable and unstable 
equilibrium positions. The switching forces can be determined by the magnitude of the maximum and minimum of the curve.

The fully compliant bistable mechanism (FCBM) contains no pin joints, gaining all motion from the deflection of flexible members [1]. The FCBM uses a central shuttle to constrain its motion to linear displacement. The FCBM topology used in this research was presented by Wittwer et al. [6] as shown in Figure 2.

Qiu [7] describes the advantages of a centrally clamped parallel beam bistable mechanism. Two parallel beams are connected in the center to insure that the buckling of the bistable mechanism is symmetric. This symmetric buckling mode can also be accomplished by the use of multiple sets of legs with a central shuttle (as in the FCBM). The shuttle acts as a central clamp, allowing the legs to move in parallel.

Variability in performance is introduced in micro mechanisms through joint clearances, friction forces, stiction, dimensional variations, and uncertain material properties [8]. Variability due to joint clearances can be eliminated by using a fully compliant bistable mechanism, but the behavior of the device shown in Figure 2 is still subject to variation in beam widths and residual stress [6]. The FCBM can be fabricated in a plane, making it practical for MEMS applications in which silicon or polysilicon is used as the structural material. Both silicon and polysilicon have been shown to be piezoresistive materials.

Piezoresistivity describes an effect in which the bulk resistivity is influenced by the mechanical stress applied to the material. This effect in silicon was described by Smith [9]. Semiconductor strain gauges utilizing this material property are two orders of magnitude more sensitive than metal gauges [10]. Changes in conductivity under stress are attributed to the 
raising and lowering of conduction band minima [11]. Warping of the band structure is also present in p-type silicon, yielding both a transfer of carriers and a change in effective mass [12].

Mathematical models have been developed to characterize changes in resistivity [13]. French and Evans developed a model, including the effects of grain boundaries, which describes the piezoresistive behavior of polysilicon [12] [14] [15].

Pressure sensors are a common application for piezoresistivity in polysilicon [10] [11] [16] [17]. Pressure sensing membranes are used in combination with specially-doped resistive areas. The effect of piezoresistivity has also been used to detect acoustic waves in a microphone [18] and to detect forces in the cantilevers of atomic force microscopes [19]. In these applications, resistors are deposited on top of the structure and are in either compression or tension, depending on the direction of membrane deflection. In contrast, this work uses the entire mechanism as the resistive element, and in-plane motions are being measured instead of out-ofplane deflections.

Piezoresistivity has been used to determine the position of a thermomechanical in-plane microactuator (TIM) [20] [21]. Displacement measurements were captured by piezoresistive position sensing and used in feedback control of the TIM. The measurement setup is shown in Figure 3. Sensing beams were attached to the shuttle of the thermal actuator. The sensing beams then acted as resistors in a Wheatstone bridge, with both the sensing legs and the other resistors reflecting the geometry of a single set of actuator legs. As the TIM changed position, it deflected the sensing beam. This deflection induced stress, which changed the resistance of the sensing beam and the voltage output of the bridge. The position was determined by the change in voltage, and the input signal to the TIM could be adjusted. 
While the piezoresistive properties of mono crystalline silicon are generally wellunderstood and well-documented, polysilicon properties are more obscure and are complicated by process dependencies and variations. Values for the material bulk resistivity, $\rho$, and the piezoresistive matrix coefficients, $\pi_{\mathrm{ij}}$, are used for finite element modeling. The matrix coefficients relate the piezoresistivity to the stress along different directions in the material. Symmetry conditions reduce the matrix to include only $\pi_{11}, \pi_{12}$, and $\pi_{44}$. The matrix coefficients are sensitive to crystal orientation, doping type, doping level, and operating temperature [22]. Smith [9] reports the resistivity and matrix coefficients for mono crystalline n- and p-type silicon. It is predicted that polysilicon has a large, negative $\pi_{11}$ value, a $\pi_{12}$ value of the opposite sign and half the magnitude, while $\pi_{44}$ is predicted to be zero [11]. French and Evans [12] report averaged $\pi_{\mathrm{l}}$ and $\pi_{\mathrm{t}}$ (longitudinal and transverse) values for polysilicon over 5 planes plus random orientation structure. Gridchin and Lubimsky [23] provide formulas to calculate polysilicon matrix coefficients for different crystal orientations from the mono crystalline values.

Accurate values for $\pi_{11}, \pi_{12}$, and $\pi_{44}$ are not currently available for the polysilicon used in this work because these material properties have not been accurately characterized. However, the primary objective of this work is to demonstrate that bistable mechanism state can be determined using the piezoresistive effect. The models developed in the work are meant to predict trends, and the piezoresistive properties published by Smith [9] for n-type silicon are found to be adequate for this purpose. It was shown that the modeling results with these values show the important trends and give a conservative estimate of most experimental measurements. The values used for the modeling in this paper are listed in Table 1.

Piezoresistive position sensing is advantageous due to the small size of the sensing mechanism. Capacitive sensing is commonly used in sensing changes in mechanism position 
(e.g. as seen in accelerometers). Hälg [24] designed a bistable beam with capacitive sensing capabilities. The beam was electrostatically actuated and would remain in either position for an indefinite amount of time. A limitation of capacitive sensing is the very small change in capacitance typical in MEMS applications that is difficult to measure. Piezoresistive sensing uses much less space and can produce greater differences in signal when the bistable mechanism switches position. Data collection can be achieved using electrical probes or wire-bonding circuitry as opposed to capacitive sensing, which usually requires on-chip circuitry. These capabilities make piezoresistive position sensing a feasible and promising alternative to capacitive sensing.

\section{Direct Resistance Measurement}

The simplest possible configuration is to use the flexible elements of the bistable mechanism as the sensing elements, without integration with a Wheatstone bridge or other device. This section investigates the ability to detect device position without the use of a Wheatstone bridge by testing devices fabricated using two different surface micromachining processes, the SUMMiT IV process and MUMPs. A single bistable mechanism, fabricated using the SUMMiT IV process, was tested for a measurable resistance change between stable positions. The design parameters for the SUMMiT IV fully compliant bistable micromechanism are shown in Figure 4. Values and design variables for the bistable micromechanism are listed in Table 2. The values represent dimensions as drawn for manufacturing using the SUMMiT IV process (an additional $0.2 \mathrm{~mm}$ should be subtracted from the three width dimensions due to etch bias). The bistable mechanism tested had identical dimensions to the mechanisms later tested in bridge configurations. One mechanism was tested with five repetitions, and remote sensing was used to 
ensure accuracy of the applied current. Five levels of current were applied to the mechanism, and the voltage was measured in both positions. The results of this testing are shown in Table 3 .

Bistable mechanisms produced in MUMPs [25] were also tested to determine the magnitude of the resistance change associated with the toggling of the mechanism's position. The design parameters for the MUMPs fully compliant bistable micromechanism are shown in Figure 5. In addition to having different dimensions because of different process design rules, the MUMPs configuration is slightly different than the SUMMiT IV configuration in that the connection to the substrate occurs through an additional flexible member (see left of Figure 5). Values and design variables for the bistable micromechanism are listed in Table 4. Remote sensing was again used to ensure that the mechanism experienced the desired applied current. Three identical bistable mechanisms were measured on two separate die. Each mechanism was measured five times in both stable positions. The measurements for the MUMPs bistable mechanisms are listed in Table 5. The results for the MUMPs and SUMMiT configurations must be evaluated independently because they were fabricated by different processes, made with different material properties, and used different bistable mechanism designs. Detectable voltage changes were recorded for both mechanisms independent of bridge integration.

While the resistance of the SUMMiT device remained constant throughout all input currents (calculated by dividing output voltages by input currents), a nonlinear trend (Figure 6) occurred in the resistance of the MUMPs device as the source current increased. The maximum standard deviation associated with these measurements was $1.1 \Omega$, demonstrating that the nonlinearity of the data points is larger than the standard deviation of the measurements. The nonlinearity of the mechanism's resistance could be attributed to the temperature change of the material as described by Gad-el-Hak [22]. This is reasonable, because a similar topology is used 
for thermal actuators, where resistivity is known to be affected by temperature [26]. This section demonstrates the feasibility of piezoresistive sensing of bistable mechanism state using the flexible members of the bistable mechanism as sensing elements. This simple approach leads to the investigation of devices integrated in a Wheatstone bridge configuration, as described next.

\section{System Device Design}

Bistable mechanisms were fabricated (SUMMiT IV) in Wheatstone bridge configurations, where mechanisms with the same dimensions described in Table 2 were used as resistors in the bridges. Figure 7 displays the four fabricated configurations with assigned labels A through D.

Configuration A (Figure 7 (a)) uses a quarter bridge configuration with all four resistors made of identical bistable mechanisms. The identical mechanisms were used to ensure that the Wheatstone bridge was balanced. The balanced bridge is designed to yield an output voltage of zero while the mechanism is in the first stable position, because the bridge will be symmetric. When the mechanism changes position, the bridge will no longer be balanced, and the output voltage will be non-zero.

Figure 7 (d) (configuration D) shows a quarter bridge with two mechanisms and two beams. The beams were designed to have resistances similar to that of the bistable mechanism. The lower mechanism is physically stopped to prevent deflection under residual stress.

Similar to configuration D, configuration B (Figure 7 (b)) uses two mechanisms and two beam resistors. The shuttles of the two mechanisms are connected in configuration $\mathrm{B}$, allowing both mechanisms to toggle positions simultaneously. The potential of yielding a higher change in resistance between the two positions exists due to the resistive changes in two mechanisms, but electrical connection of the two shuttles alters the circuitry of the bridge. 
A quarter bridge with three beam elements is shown in Figure 7 (c) (configuration C). This configuration could be designed to be the most compact, with stacked beams manufactured in different layers.

The dimensions of the beam resistors for each configuration are listed in Table 6, where configuration $\mathrm{C}$ has values for the vertical and horizontal beam resistors. Each of these configurations was tested to determine the effects of the different Wheatstone bridge architectures.

Wheatstone bridges are circuits designed to sense small changes in resistance (Figure 8). An excitation voltage is applied, and an output voltage corresponding to the resistance change is measured. For a balanced bridge $(R 1 / R 2=R 3 / R 4)$, the output voltage will be zero for any input voltage. Balanced bridges mitigate temperature varying effects because all resistors in the bridge heat up at the same rate. The equation describing the output voltage is

$$
V o=\left(\frac{R 1}{R 1+R 2}-\frac{R 3}{R 3+R 4}\right) V i
$$

where $V o$ is the output voltage, and $V i$ is the excitation voltage. Quarter-bridge configurations were used in this research, where the bistable mechanism is the variable resistor. A change in $R 1$ (the resistance of the bistable mechanism) will change to some new value, $R l^{\prime}$, where $R 1^{\prime}=R 1+\delta R$. The output from the bridge then is

$$
V o=\left(\frac{R 1^{\prime} R 4-R 3 R 2}{\left(R 1^{\prime}+R 2\right)(R 3+R 4)}\right) V i
$$

For a bridge where all the resistors are nominally equivalent (i.e. $R 1=R 2=R 3=R 4=R$ ), quarter bridge circuit, the voltage change can be simplified to contain a single resistance, $R$, such that 


$$
V o=\frac{\Delta R}{4 R} V i
$$

Wheatstone bridges are used in this research in order to detect small changes in the resistance of a bistable mechanism. The resistance of the SUMMiT bistable mechanism used in this work was measured to be $90.7 \mathrm{ohms}$ with a standard deviation of $1.03 \mathrm{ohms}$. The change in resistance detected when the mechanism toggles positions was found to be $2.0 \mathrm{ohms}$ with a standard deviation of 0.17 ohms. Table 7 shows the voltage changes predicted by equation 3 at three voltage source levels for a balanced quarter-bridge (configuration A) using these measured resistive values.

The simple model of Equation 3 was a good prediction of actual device behavior for configuration A (see Table 8). Due to balance in the bridge and identical resistors, configuration A should be insensitive to variations in temperature. Temperature changes in the bridge will be experienced equally among all resistors. Configurations $\mathrm{C}$ and $\mathrm{D}$ are quarter bridge configurations but may not be balanced due to the use of beams as resistors. These configurations will thus be more sensitive to temperature variations, and Equation 3 will be less accurate in predicting device behavior. Note that configuration B is not a Wheatstone bridge due to the connection of the central shuttles.

\section{Modeling}

Finite element analysis was performed using ANSYS to model each configuration. Twodimensional, coupled-field, piezoresistive elements (PLANE223) were used to predict the structural and piezoresistive properties of the polysilicon devices. All four layouts were modeled in their bridge configurations, with excitation voltages applied and output voltages measured. The results for all configurations were similar, with the results of configuration $\mathrm{C}$ shown in 
Figure 9. Voltage changes for three source voltages are shown as solid lines. The forcedisplacement plot (dashed line) of the bistable mechanism is displayed on the secondary axis for position reference. The vertical line indicates the position of the physical stop used to hold the second stable position at a state of higher stress. The importance of the stop can be seen by observing the small voltage changes when the mechanism reaches its natural, second stable equilibrium position (SSP). Experimental data reflects the point where each voltage curve intersects the vertical line, i.e. the voltage change between the as-fabricated position and the switched, stopped position.

\section{Testing and Results}

Ten different modules were tested, with one instance of each configuration per module. Seven of the modules were tested using probes (module numbers 1-7), while three modules were wire-bonded to obtain better electrical contacts (numbers 8-10).

\subsection{Probe Testing}

Two Keithley 2400 Digital SourceMeters were used to power the bridge and measure the output voltage utilizing seven probes. The first SourceMeter was used exclusively to apply an accurate voltage to the bridge. Four probes were used in the four-sense mode (remote sensing) to power the bridge. Two probes apply the source voltage, while two neighboring probes read the voltage to insure the application of the desired voltage level. When the source voltage is detected to vary from the desired level, the SourceMeter increases or decreases the applied power to insure that the device experiences the correct voltage. The wires connecting the power source to the probes and the interface between the probes and the bond pads introduce resistive losses into the system. This is especially important with devices fabricated using the SUMMiT IV process, 
because bond pads are not metallized. The four-wire sense mode mitigates the effects of resistive losses associated with contact pad area (including effects such as tip diameter, angle of descent, etc.) as well as the surface of contact (being clean from debris and oxidation, uniform texture), and other losses (such as that due to cable lengths, device interconnects), and facilitates the accurate application of the excitation voltage. The method reduces much of the error in the measurement, but cannot guarantee to eliminate all of it. The second SourceMeter is used as a voltmeter to measure the output voltage of the bridge. A seventh probe is used to manually actuate the mechanism between its two stable positions. This setup is shown in Figure 10 for configuration B. A similar setup was used with other configurations. Each configuration was tested at three excitation voltages: $0.5,1.0$, and 1.5 volts. Three measurements were taken with the bistable mechanism in its first position (one at each excitation voltage). The mechanism was toggled, and three more measurements were recorded in the second stable position. This sequence was repeated five times for each mechanism across the seven probe-tested modules. The power source was turned off between each measurement, and the probes adjusted to optimize the output signal.

The non-metallized bond pads of the tested devices limited the precision of the measurements. The output on the voltmeter would converge on a value after the probes were adjusted to yield better contacts. Once the output converged, the results were repeatable. Measurements were taken with 0.0001 volt precision.

\subsection{Wire-Bonded Testing}

In order to reduce measurement error due to contact resistance and other resistive losses, three modules were wire bonded. Aluminum wire was used to bond the pads to the module carrier. The procedures described for probe testing were used to obtain measurements, but four- 
wire remote sensing could not be used. The same three excitation voltages were used, and each mechanism was measured five separate times in both positions at each excitation voltage. As expected, the output voltage was very steady, showing no signs of deviation. The use of wirebonding also allowed measurements to be taken with an additional decimal place of accuracy.

\subsection{Results}

Each configuration experienced a measurable change in voltage when the bistable mechanism position was toggled. Measured average voltage changes, standard deviations, and the model predictions $\left(\Delta \mathrm{V}_{\mathrm{M}}\right)$ are listed in Table 8 for each configuration and excitation voltage level. Data is missing for modules where devices were damaged. Configuration B showed the largest change in voltage between the two positions, while all configurations yielded consistent results with small variation. Configurations $C$ and D had very similar results, but exhibited the smallest change in output voltage. Intermediate changes were experienced by configuration A. The measurements of the wire-bonded parts exhibited smaller variation with voltage changes similar to those seen with the probe-tested modules. All voltage changes are greater than $2.2 \mathrm{mV}$, which can be easily measured.

The models for configurations B, C, and D under-predict the voltage change, whereas the model over-predicts the signal for configuration A. The models for configurations C and D predict approximately $80 \%$ of the experimentally- obtained voltage change. The discrepancy between the model predictions and the measured values is believed to be primarily due to the inaccurate piezoresistive coefficients used in the models. 


\section{Conclusions}

Resistance changes of a micro bistable mechanism as it toggles positions can be made to be detectable and repeatable. A $1.5 \mathrm{~V}$ excitation voltage produced up to an $11.3 \mathrm{mV}$ difference between the output voltages at each of the two stable positions. Ensuring that adequate compressive stresses are in the mechanism in its second stable state is one method of insuring a measurable change between positions. This phenomenon can be modeled using finite element analysis, and the resulting trends may be used to enhance device design.

By using the mechanism as a sensing element in a Wheatstone bridge, on-chip sensing can be integrated into the system. Low power is needed to sense the state of the mechanism $(2.8$ $\mathrm{mW}$ for a $0.5 \mathrm{~V}$ input), and the bridge configuration provides convenient, repeatable results. Time-varying factors are also eliminated by the use of the Wheatstone bridge.

A significant advantage of using piezoresistive properties rather than electrical contacts to detect state is that the reliability problems associated with electrical contacts can be eliminated, because the completion of an electrical circuit is no longer needed to determine the device position. Device design is thus simplified because no consideration must be taken for contact positioning, and device reliability is dramatically increased by eliminating the dependence on problematic micro contacts.

Possible applications for the bistable mechanism with piezoresistive position sensing include mechanical nonvolatile memory and high-acceleration sensing arrays. Bistable mechanisms are well-suited for mechanical nonvolatile memory applications, because they remain in position without any input power. Acceleration thresholds can be calculated using the mechanism switching force and the mass of the central shuttle. An array of mechanisms can be designed with varying acceleration thresholds. This array can then be queried following an 
impact to determine the magnitude of the experienced acceleration. Piezoresistivity may also be used to measure the dynamic response of a device by taking measurements as the device toggles.

\section{Acknowledgements}

The authors wish to thank BlockMEMS for supporting device fabrication, and Sandia National Labs for their support of the testing through the NSF-Sandia Collaborative Research Program. This research was funded by National Science Foundation grant CMS-0428532. 


\section{References}

[1] Parkinson, M.B., Jensen, B.D., and Roach, G.M., 2000, “Optimization-Based Design of a Fully-Compliant Bistable Micromechanism," 2000 ASME Design Engineering Technical Conferences and Computer and Information in Engineering Conference, Sept. 10-13, 2000, Baltimore, Maryland.

[2] Foulds, I., Trinh, M., Hu, S., Liao, S., Johnstone, R., Parameswaran, M., 2002, “A Surface Micromachined Bistable Switch," IEEE CCECE2002, Vol. 1, pp. 465-469.

[3] Wagner, B., Quenzer, H.J., Hoerschelmann, S., Lisec, T., Juerss, M., 1996, "Bistable Microvalve with Pneumatically Coupled Membranes," 9th International IEEE Conference, pp. 384-388.

[4] Qiu, J., Lang, J.H., Slocum, A. H., Strumpler, R., 2003, “A High-Current Electrothermal Bistable MEMS Relay," 16th Internation IEEE Conference on Micro Electro Mechanical Systems (MEMS), pp. 64-67.

[5] Opdahl, P. G., Jensen, B.D., and Howell, L.L., 1998, "An Investigation into Compliant Bistable Mechanisms," Proceedings of the 1998 ASME Design Engineering Technical Conferences, DETC09/DAC-3763.

[6] Wittwer, J.W., Baker, M.S., and Howell, L.L., "Robust Design and Model Validation of Nonlinear Compliant Micromechanisms," Journal of Microelectromechanical Systems, Vol. 15, No. 1, pp. 33-41, 2006.

[7] Qui, J., Lang, J.H., Slocum, A.H., 2001, “A Centrally-Clamped Parallel-Beam Bistable MEMS Mechanism," 14th International IEEE Conference, pp. 353-356.

[8] Wittwer, J.W., Wait, S.M., Howell, L.L., Cherry, M.S., 2002, "Predicting the performance of a bistable micro mechanism using design-stage uncertainty analysis," ASME, MicroElectromechanical Systems Division Publication (MEMS), pp. 127-134.

[9] Smith, C.S., 1954, "Piezoresistance Effect in Germanium and Silicon," Physical Review, 94,pp. 42-49.

[10] Elgamel, H.E., 1995, "Closed-form expressions for the relationships between stress, diaphragm deflection, and resistance change with pressure in silicon piezoresistive pressure sensors," Sensors and Actuators, 50, pp. 17-22.

[11] Eaton, W.P., 1997, "Surface Micromachined Pressure Sensors," Ph.D. Dissertation, University of New Mexico, Albuquerque, NM.

[12] French, P.J., Evans, A.G.R., 1989, "Piezoresistance in Polysilicon and its Applications to Strain Gauges," Solid State Electronics, Vol. 32, No. 1, pp. 1-10. 
[13] Wymyslowski, A., Santo-Zarnki, M., Friedel, K., Belavic, D., 2004, "Numerical simulation and Experimental Verification of the Piezoresistivity Phenomenon for the Printed ThickFilm Piezoresistors," Proceedings of the 5th International Conference on Thermal and Mechanical Simulation and Experiments in Microelectronics and Microsystems, pp. 359366.

[14] French, P.J., Evans, A.G.R., 1985, "Polycrystalline silicon strain sensors," Sensors and Actuators, 8, pp. 219-225.

[15] French, P.J., Evans, A.G.R., 1986, "Polycrystalline silicon as a strain gauge material," Journal of Physics E: Scientific Instruments, 19, pp. 1055-1058.

[16] Burns, D., 1988, "Micromechanics of Integrated Sensors and the Planar Processed Pressure Transducer," Ph.D. dissertation, University of Wisconsin-Madison.

[17] Eaton, W.P., Smith, J.H., "Planar Surface-Micromachined Pressure Sensor with a SubSurface, Embedded Reference Pressure Cavity," Proceeding of the SPIE, Vol. 2882.

[18] Schellin, R., Hess, G., 1992, "Silicon subminiature microphone based on piezoresistive polysilicon strain gauges," Sensors and Actuators, v. 32, n. 1-3, pp. 555-559.

[19] Liang, Y., Kenny, T., 1998, "Mechanical optimization of a dual-axis piezoresistive force sensor," ASME, DSC Division, MEMS v. 66, pp. 389-394.

[20] Messenger, R.K., 2004, Modeling and Control of Surface Micromachined Thermal Actuators, M.S. Thesis, Brigham Young University, Provo, UT.

[21] Messenger, R.K., McLain, T.W., Howell, L.L., 2004, "Feedback Control of a Thermomechanical Inplane Microactuator Using PiezoResistive Displacement Sensing," Proceeding of 2004 ASME International Mechanical Engineering Congress, IMECE200459810.

[22] Gad-el-Hak, M., 2001, The MEMS Handbook, CRC Press, Boca Raton, FL.

[23] Gridchin, V.A., Lubimsky, V.M., 2003, "Phenomonological Model of the Piezoresistive Effect in Polysilicon Films," Russian Microelectronics, Vol. 32, No. 4, pp. 205-213.

[24] Hälg, B., 1990, “On a Micro-Electro-Mechanical Nonvolatile Memory Cell,” Proceedings of IEEE Micro-Electro-Mechanical Systems, An Investigation on Micro Structures, Sensors, Actuators, Machines and Robots, pp. 172-176.

[25] Koester, D.A., Mahadevan, R., Hardy, B., Markus, K.W., 2001, MUMPs Design Handbook, Research Triangle Park, N.C.: Cronos Integrated Microsystems. 
[26] Lott, C.D., McLain, T.W., Harb, J.N., Howell, L.L., 2001, "Thermal Modeling of a Surface-Micromachined Linear Thermal Actuator," Modeling and Simulation of Microsystems, pp. 370-373. 
Table 1. Piezoresistive values used for FEA modeling [9].

\begin{tabular}{ll}
\hline$\rho(\mathrm{T} \Omega \mu \mathrm{m})$ & $11.7 \mathrm{e}-8$ \\
\hline$\pi_{11}(\mathrm{MPa})^{-1}$ & $-102.2 \mathrm{e}-5$ \\
\hline$\pi_{12}(\mathrm{MPa})^{-1}$ & $53.4 \mathrm{e}-5$ \\
\hline$\pi_{44}(\mathrm{MPa})^{-1}$ & $-13.6 \mathrm{e}-5$ \\
\hline
\end{tabular}

Table 2. Design variables and values.

\begin{tabular}{cc}
\hline Variable & Value \\
\hline$l_{l}(\mu \mathrm{m})$ & 21.6 \\
\hline$w_{l}(\mu \mathrm{m})$ & 1.7 \\
\hline$\theta_{l}(\mathrm{deg})$. & 7.0161 \\
\hline$l_{r}(\mu \mathrm{m})$ & 80.0 \\
\hline$w_{r}(\mu \mathrm{m})$ & 6.2 \\
\hline$\theta_{r}(\mathrm{deg})$. & 2.167 \\
\hline$l_{2}(\mu \mathrm{m})$ & 21.6 \\
\hline$w_{2}(\mu \mathrm{m})$ & 1.65 \\
\hline$\theta_{2}(\mathrm{deg})$. & 2.039 \\
\hline
\end{tabular}

Table 3. SUMMiT voltage changes for mechanism without Wheatstone bridge.

SUMMiT Mechanism

\begin{tabular}{ccc}
$\mathbf{I}_{\text {in }}(\mathbf{m A})$ & $\Delta \mathbf{V}(\mathbf{m V})$ & Std. Dev. $(\mathbf{m V})$ \\
\hline 0.5 & 0.94 & 0.15 \\
\hline 1.0 & 2.0 & 0.11 \\
\hline 1.5 & 3.0 & 0.16 \\
\hline 2.0 & 4.2 & 0.13 \\
\hline 2.5 & 5.3 & 0.26 \\
\hline
\end{tabular}


Table 4. MUMPs design variables and values.

\begin{tabular}{cc}
\hline Variable & Value \\
\hline$l_{l}(\mu \mathrm{m})$ & 95.7 \\
\hline$w_{l}(\mu \mathrm{m})$ & 2.5 \\
\hline$\theta_{1}($ deg. $)$ & 6.9 \\
\hline$r_{10}(\mu \mathrm{m})$ & 145.5 \\
\hline$\theta_{0}($ deg. $)$ & 6.1 \\
\hline$l_{2}(\mu \mathrm{m})$ & 77.3 \\
\hline$w_{2}(\mu \mathrm{m})$ & 2.5 \\
\hline$\theta_{2}(\mathrm{deg})$. & 7.1 \\
\hline$l_{k}(\mu \mathrm{m})$ & 64 \\
\hline$w_{k}(\mu \mathrm{m})$ & 3.5 \\
\hline
\end{tabular}

Table 5. MUMPs voltage changes for mechanisms without Wheatstone bridge.

\begin{tabular}{ccc}
\hline & \multicolumn{2}{c}{ MUMPs Mechanisms } \\
$\mathbf{I}_{\text {in }}(\mathbf{m A})$ & $\Delta \mathbf{V}(\mathbf{m V})$ & Std. Dev. $(\mathbf{m V})$ \\
\hline 0.5 & 4.9 & 0.25 \\
\hline 1.0 & 9.7 & 0.48 \\
\hline 1.5 & 14 & 0.91 \\
\hline 2.0 & 18 & 1.7 \\
\hline 2.5 & 21 & 3.0 \\
\hline
\end{tabular}


Table 6. Beam resistor dimensions.

\begin{tabular}{cccc}
\hline & $\begin{array}{c}\text { Width } \\
(\mu \mathbf{m})\end{array}$ & $\begin{array}{c}\text { Length } \\
(\mu \mathbf{m})\end{array}$ & $\begin{array}{c}\text { Thickness } \\
(\mu \mathbf{m})\end{array}$ \\
\hline Configuration B & 9.367 & 26.0 & 0.3 \\
\hline Configuration C (1) & 2.989 & 16.0 & 0.3 \\
\hline Configuration C (2) & 4.416 & 265.028 & 2.8 \\
\hline Configuration D & 2.989 & 16.0 & 0.3 \\
\hline
\end{tabular}

Table 7. Predicted voltage changes for configuration A.

\begin{tabular}{cc}
\hline $\mathbf{V}_{\mathbf{s}}($ Volts $)$ & $\Delta \mathbf{V}(\mathbf{m V})$ \\
\hline 0.5 & 2.77 \\
\hline 1.0 & 5.53 \\
\hline 1.5 & 8.30 \\
\hline
\end{tabular}

Table 8. Voltage changes, standard deviations, and model predictions (mV).

$$
\text { Configuration A Configuration B Configuration } C \text { Configuration } D
$$

\begin{tabular}{cccccccccccccc}
$\mathbf{V}_{\mathbf{s}}$ (Volts) & $\Delta \mathbf{V}$ & Std. Dev. & $\Delta \mathbf{V}_{\mathbf{M}}$ & $\Delta \mathbf{V}$ & Std. Dev. & $\Delta \mathbf{V}_{\mathbf{M}}$ & $\Delta \mathbf{V}$ & Std. Dev. $\Delta \mathbf{V}_{\mathbf{M}} \Delta \mathbf{V}$ & Std. Dev. & $\Delta \mathbf{V}_{\mathbf{M}}$ \\
\hline 0.5 & 2.8 & 0.18 & 4.5 & 3.6 & 0.20 & 1.9 & 2.2 & 0.11 & 1.7 & 2.2 & 0.11 & 1.7 \\
\hline 1.0 & 5.9 & 0.19 & 9.0 & 7.4 & 0.32 & 3.7 & 4.5 & 0.18 & 3.4 & 4.4 & 0.22 & 3.4 \\
\hline 1.5 & 9.1 & 0.16 & 13 & 11 & 0.46 & 5.6 & 6.7 & 0.32 & 5.2 & 6.6 & 0.31 & 5.2 \\
\hline
\end{tabular}




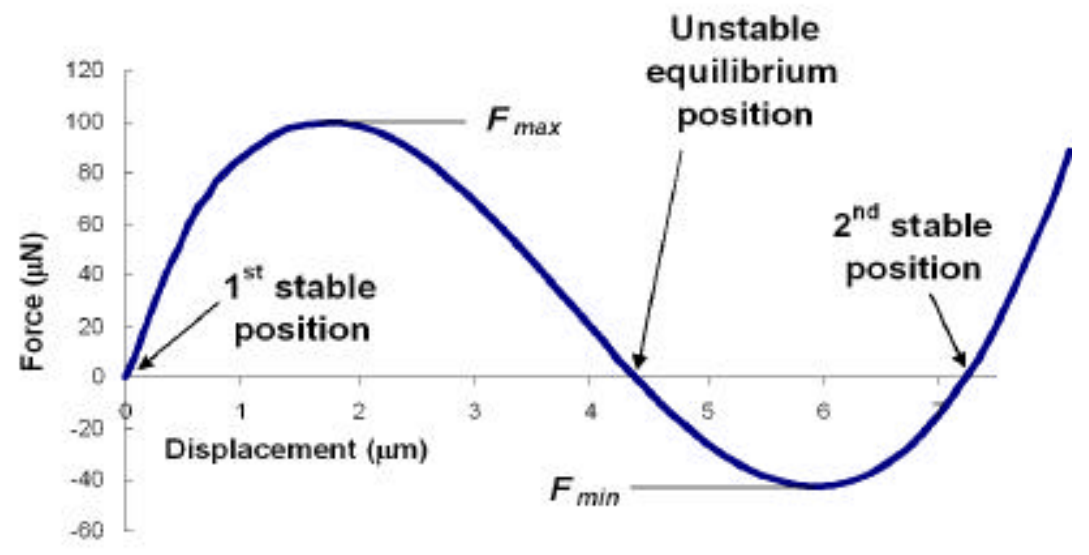

Figure 1. Typical force vs. displacement curve of a bistable mechanism.
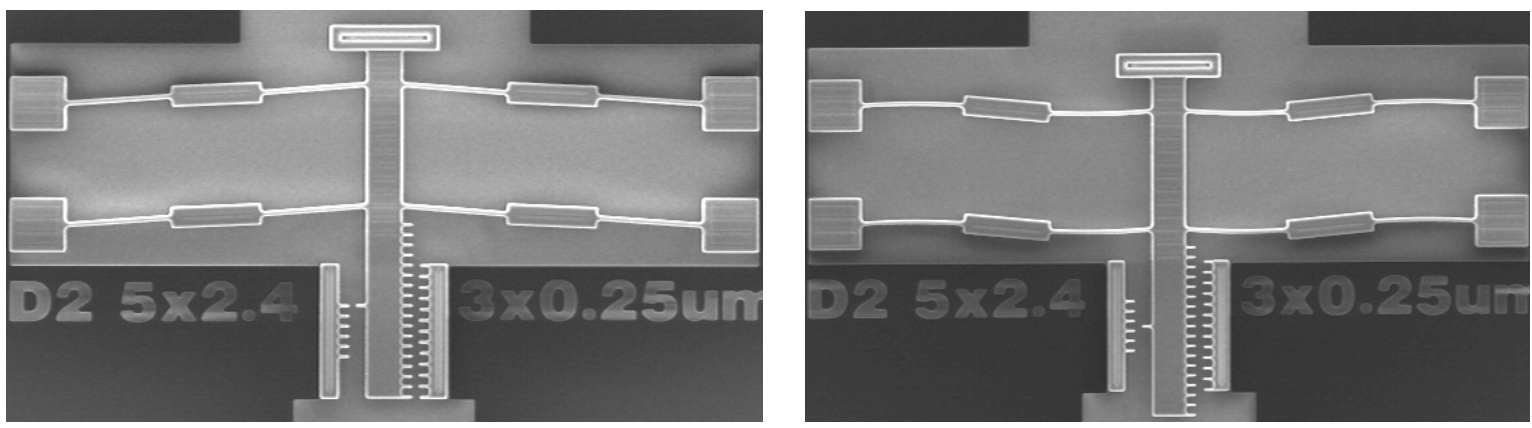

Figure 2. Fully compliant micro bistable mechanism in first (left) and second (right) stable equilibrium positions [6].

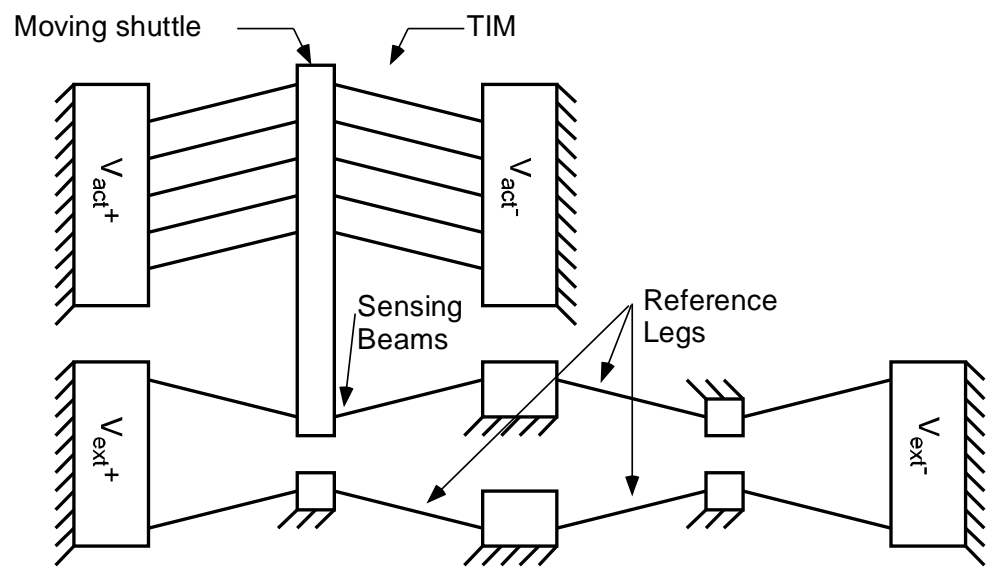

Figure 3. Piezoresistive position sensing setup for feedback control of a thermal actuator $\lceil 211$. 


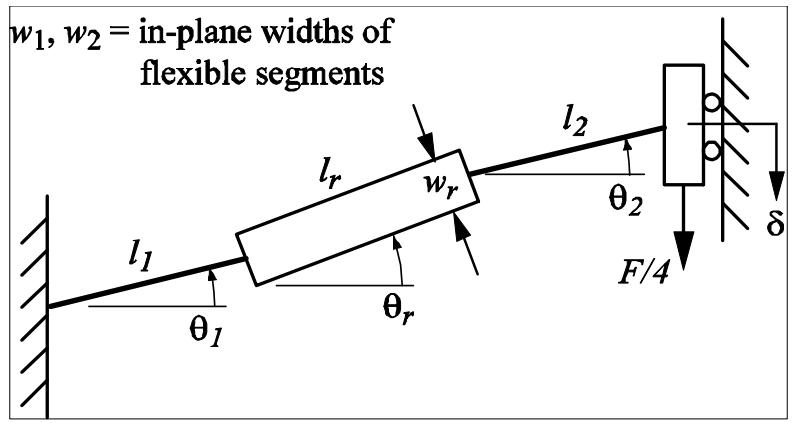

Figure 4. Design parameters of the quarter-model of a SUMMiT IV fully compliant bistable mechanism $\lceil 61$.

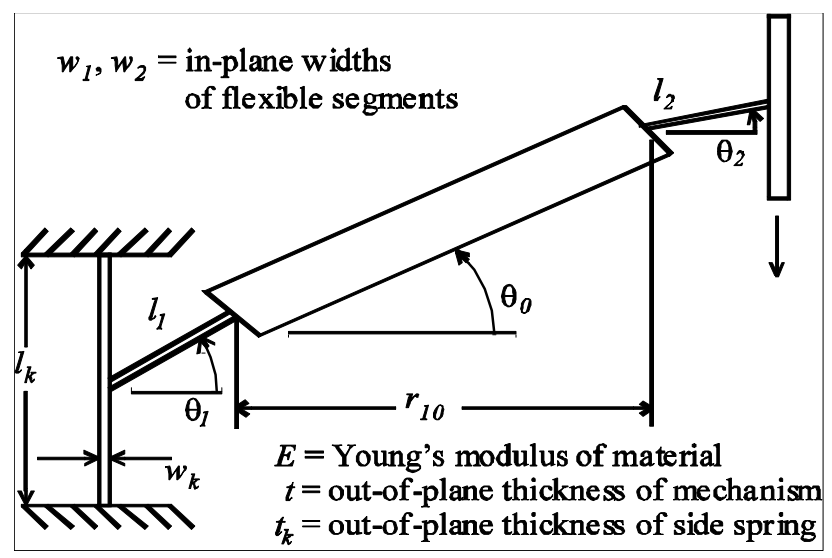

Figure 5. Design parameterization of MUMPs fully compliant bistable mechanism [1]. Note the similarity with the device of Figure 4, the primary difference being the flexible connection a the loft side

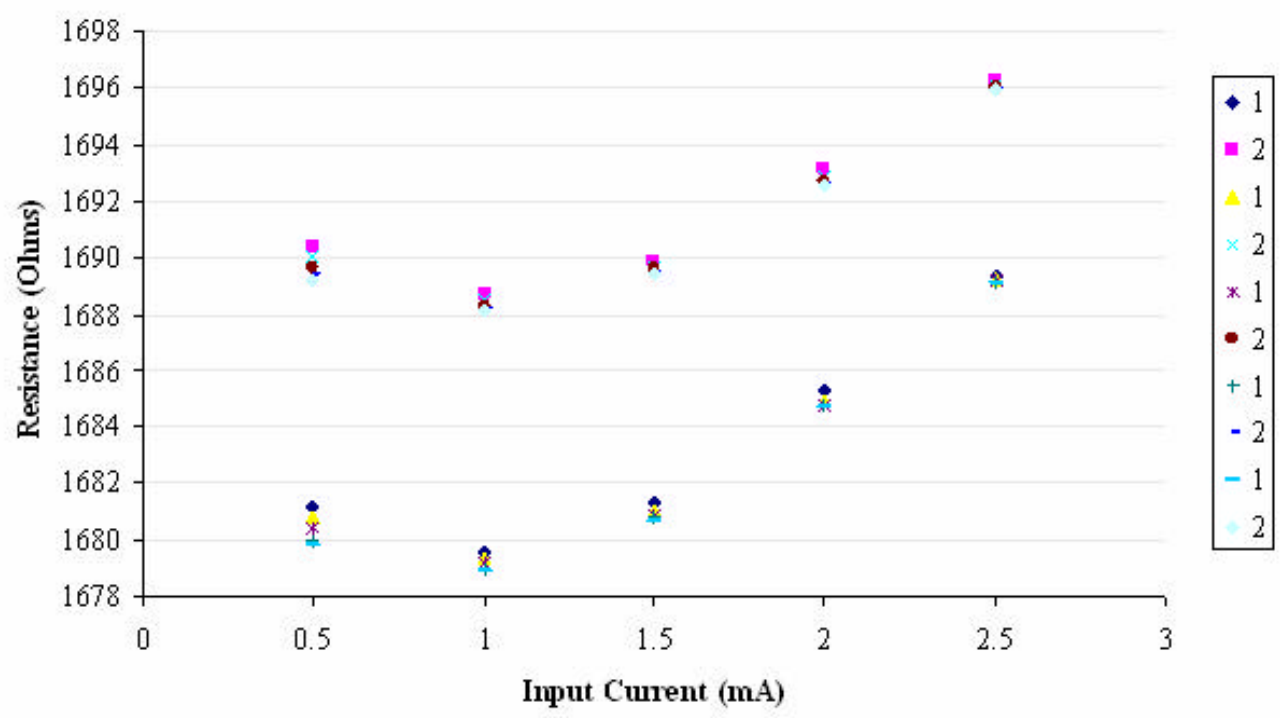

Figure 6. Resistance change with current of MUMPs bistable mechanism. 

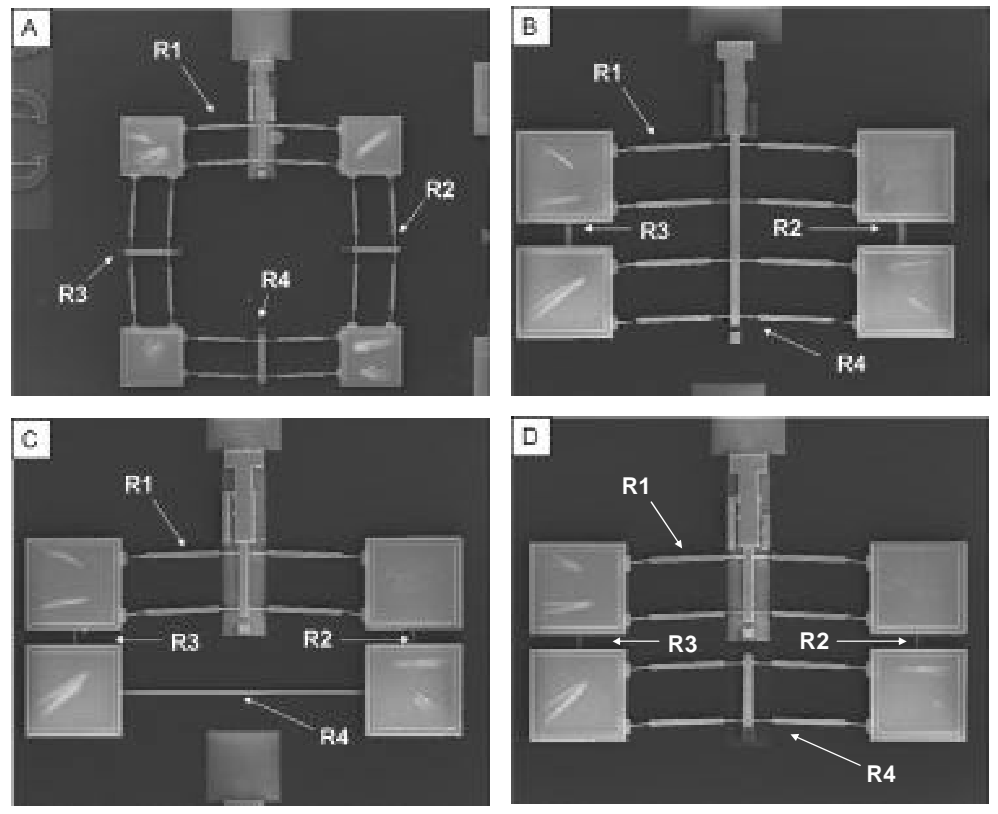

Figure 7. SEM micrograph of four bistable mechanism bridge configurations (Configuration A is shown at a different magnification than the other configurations).

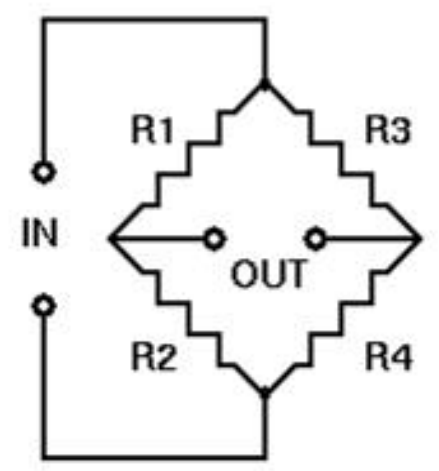

Figure 8. Wheatstone bridge schematic. 


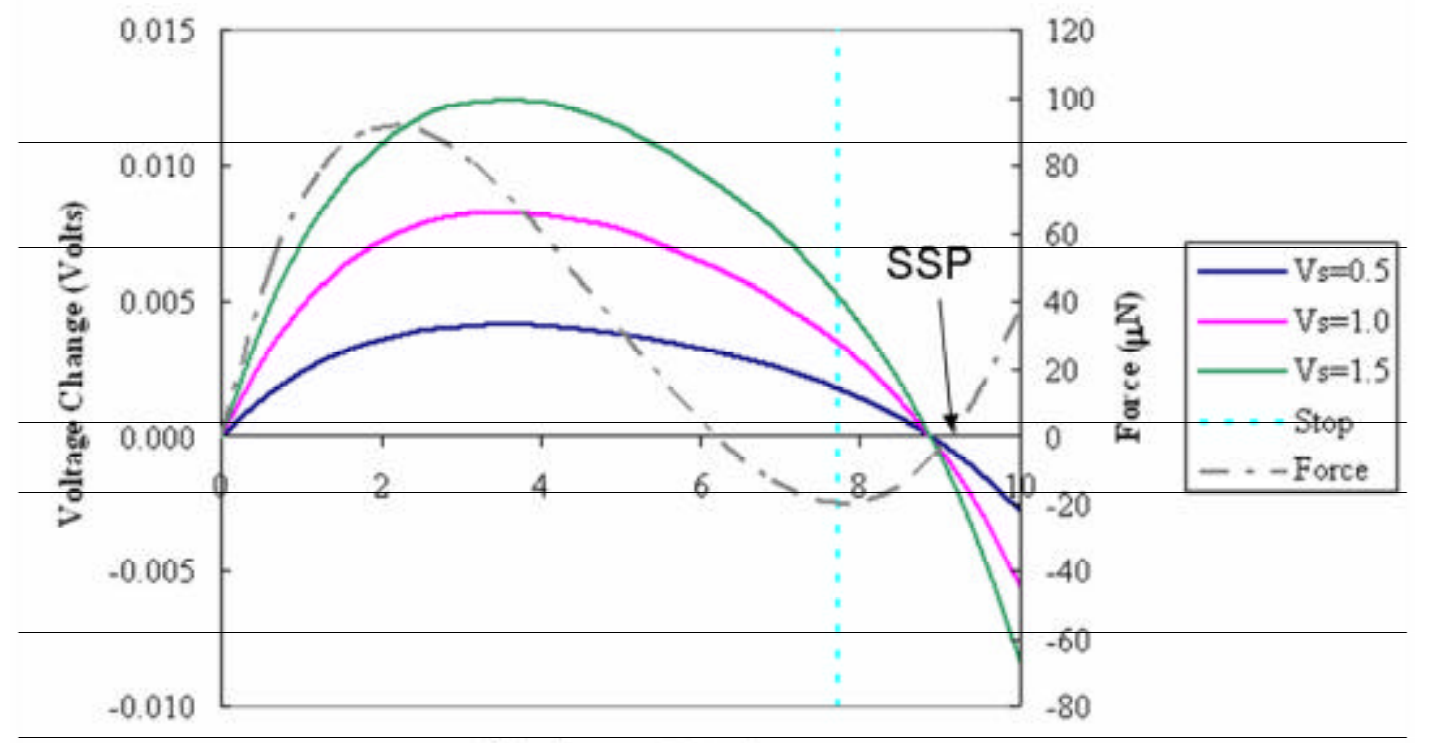

Displacement ( $\mu \mathrm{m})$

Figure 9. Configuration $\mathrm{C}$ modeling results.

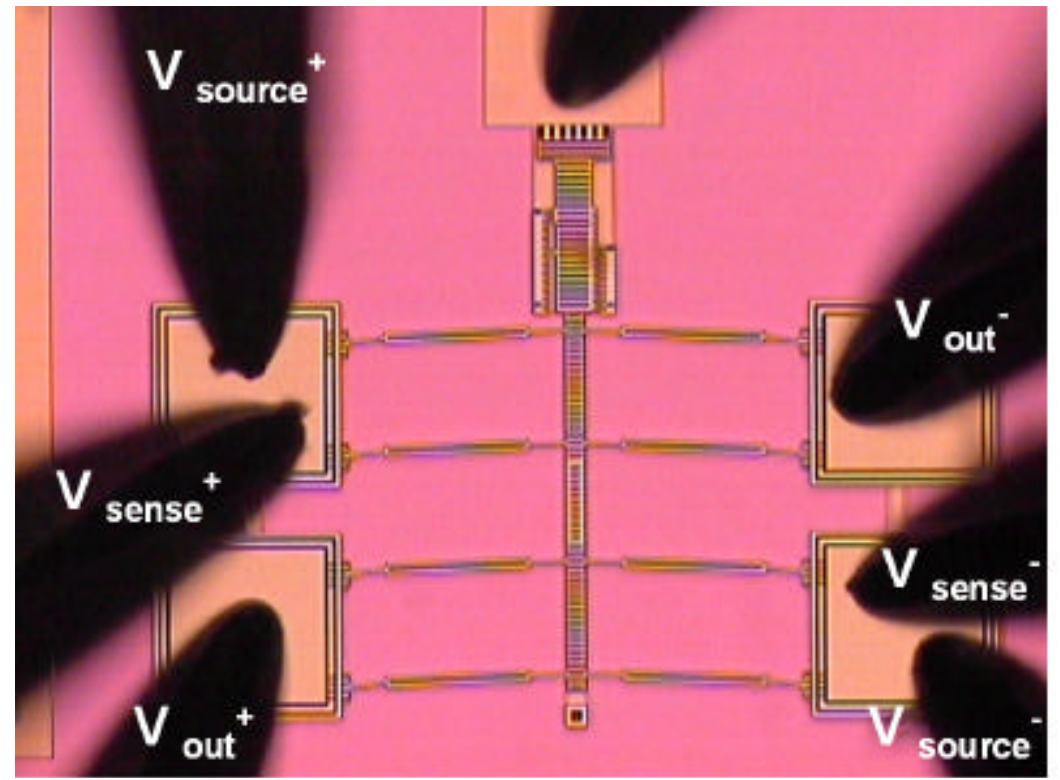

Figure 10. Probe setup for piezoresistive position testing. 